\title{
Cosmology with Clusters of Galaxies
}

\author{
Neta A. Bahcall* \\ Princeton University Observatory \\ Princeton, NJ 08544
}

December 31, 1998

\begin{abstract}
Rich clusters of galaxies, the largest virialized systems known, place some of the most powerful constraints on cosmology. I discuss below the use of clusters of galaxies in addressing two fundamental questions: What is the mass-density of the universe? and how is the mass distributed? I show that several independent methods utilizing clusters of galaxies - cluster dynamics and mass-to-light ratio, baryon fractions in clusters, and cluster evolution - all indicate the same robust result: the mass-density of the universe is low, $\Omega_{m} \simeq 0.2$, and the mass approximately traces light on large scales.
\end{abstract}

\section{Introduction}

Theoretical arguments based on standard models of inflation, as well as on the demand of no "fine tuning" of cosmological parameters, predict a flat universe with the critical density needed to just halt its expansion. The critical density, $1.9 \times 10^{-29} \mathrm{~h}^{2} \mathrm{~g} \mathrm{~cm}^{-3}$ (where $\mathrm{h}$ refers to Hubble's constant, see below), is equivalent to $\sim 10$ protons per cubic meter; this density provides the gravitational pull needed to slow down the universal expansion and will eventually bring it to a halt. So far, however, only a small fraction of the critical density has been detected, even when all the unseen dark matter in

*neta@astro.princeton.edu 
galaxy halos and clusters of galaxies is included. There is no reliable indication so far that most of the matter needed to close the universe does in fact exist. Here we show that several independent observations of clusters of galaxies all indicate that the mass density of the universe is sub-critical. These observations include the mass and mass-to-light ratio of clusters and superclusters of galaxies, the high baryon fraction observed in clusters, and the evolution of the number density of massive clusters with time; the latter method provides a powerful measure not only of the mass-density of the universe but also the amplitude of the mass fluctuations. The three independent methods- all simple and robust- yield consistent results of a low-density universe with mass approximately tracing light on large scales.

\section{Cluster Dynamics and the Mass-to-Light Ratio}

Rich clusters of galaxies are the most massive virialized objects known. Cluster masses can be directly and reliably determined using three independent methods: (i) the motion (velocity dispersion) of galaxies within clusters reflect the dynamical cluster mass, within a given radius, assuming the clusters are in hydrostatic equilibrium [1-3]; (ii) the temperature of the hot intracluster gas, like the galaxy motion, traces the cluster mass [4-6]; and (iii) gravitational lensing distortions of background galaxies can be used to directly measure the intervening cluster mass that causes the distortions [7-10]. All three independent methods yield consistent cluster masses (typically within radii of $\sim 1 \mathrm{Mpc}$ ), indicating that we can reliably determine cluster masses within the observed scatter $(\sim \pm 30 \%)$.

The simplest argument for a low density universe is based on summing up all the observed mass(associated with light to the largest possible scales) by utilizing the well-determined masses of clusters. The masses of rich clusters of galaxies range from $\sim 10^{14}$ to $10^{15} \mathrm{~h}^{-1} \mathrm{M}_{\odot}$ within $1.5 \mathrm{~h}^{-1} \mathrm{Mpc}$ radius of the cluster center (where $\mathrm{h}=\mathrm{H}_{0} / 100 \mathrm{~km} \mathrm{~s}^{-1} \mathrm{Mpc}^{-1}$ denotes Hubble's constant). When normalized by the cluster luminosity, a median mass-tolight ratio of $\mathrm{M} / \mathrm{L}_{\mathrm{B}} \simeq 300 \pm 100 \mathrm{~h}$ in solar units $\left(\mathrm{M}_{\odot} / \mathrm{L}_{\odot}\right)$ is observed for rich clusters, independent of the cluster luminosity, velocity dispersion, or other parameters $[3,11]$. $\left(\mathrm{L}_{\mathrm{B}}\right.$ is the total luminosity of the cluster in the blue band, 
corrected for internal and Galactic absorption.) When integrated over the entire observed luminosity density of the universe, this mass-to-light ratio yields a mass density of $\rho_{\mathrm{m}} \simeq 0.4 \times 10^{-29} \mathrm{~h}^{2} \mathrm{~g} \mathrm{~cm} \mathrm{~cm}^{-3}$, or a mass density ratio of $\Omega_{m}=\rho_{m} / \rho_{\text {crit }} \simeq 0.2 \pm 0.1$ (where $\rho_{\text {crit }}$ is the critical density needed to close the universe). The inferred density assumes that all galaxies exhibit the same high $\mathrm{M} / \mathrm{L}_{\mathrm{B}}$ ratio as clusters, and that mass follows light on large scales. Thus, even if all galaxies have as much mass per unit luminosity as do massive clusters, the total mass of the universe is only $\sim 20 \%$ of the critical density. If one insists on esthetic grounds that the universe has a critical density $\left(\Omega_{m}=1\right)$, then most of the mass of the universe has to be unassociated with galaxies (i.e., with light). On large scales $\left(\gtrsim 1.5 \mathrm{~h}^{-1} \mathrm{Mpc}\right.$ ) the mass has to reside in "voids" where there is no light. This would imply, for $\Omega_{m}=1$, a large bias in the distribution of mass versus light, with mass distributed considerably more diffusely than light.

Is there a strong bias in the universe, with most of the dark matter residing on large scales, well beyond galaxies and clusters? An analysis of the mass-to-light ratio of galaxies, groups, and clusters by Bahcall, Lubin and Dorman [11] suggests that there is not a large bias. The study shows that the $M / L_{B}$ ratio of galaxies increases with scale up to radii of $R \sim 0.2$ $\mathrm{h}^{-1} \mathrm{Mpc}$, due to very large dark halos around galaxies [see also 12,13]. The $\mathrm{M} / \mathrm{L}$ ratio, however, appears to flatten and remain approximately constant for groups and rich clusters from scales of $\sim 0.2$ to at least $1.5 \mathrm{~h}^{-1} \mathrm{Mpc}$ and possibly even beyond (Figure 1 ). The flattening occurs at $\mathrm{M} / \mathrm{L}_{\mathrm{B}} \simeq 200-300 \mathrm{~h}$, corresponding to $\Omega_{m} \simeq 0.2$. (An $\mathrm{M} / \mathrm{L}_{\mathrm{B}} \simeq 1350 \mathrm{~h}$ is needed for a critical density universe, $\Omega_{m}=1$.) This observation contradicts the classical belief that the relative amount of dark matter increases continuously with scale, possibly reaching $\Omega_{m}=1$ on large scales. The available data suggest that most of the dark matter may be associated with very large dark halos of galaxies and that clusters do not contain a substantial amount of additional dark matter, other than that associated with (or torn-off from) the galaxy halos, plus the hot intracluster gas. This flattening of $\mathrm{M} / \mathrm{L}$ with scale, if confirmed by further larger-scale observations, suggests that the relative amount of dark matter does not increase significantly with scale above $\sim 0.2 \mathrm{~h}^{-1} \mathrm{Mpc}$. In that case, the mass density of the universe is low, $\Omega_{m} \sim 0.2-0.3$, with no significant bias (i.e., mass approximately following light on large scales).

Recently the mass and mass-to-light ratio of a supercluster of galaxies, on a scale of $\sim 6 \mathrm{~h}^{-1} \mathrm{Mpc}$, was directly measured using observations of weak 
gravitational lensing distortion of background galaxies (Kaiser et al. [14]). The results yield a supercluster mass-to-light ratio (on $6 \mathrm{~h}^{-1} \mathrm{Mpc}$ scale) of $\mathrm{M} / \mathrm{L}_{\mathrm{B}}=280 \pm 40 \mathrm{~h}$, comparable to the mean value observed for the three individual clusters that are members of this supercluster. These results provide a powerful confirmation of the suggested flattening of $M / L_{B}(R)$ presented in Figure 1 (Bahcall et al. [11,15]). The recent results confirm that no significant amount of additional dark matter exists on large scales. The results also provide a clear illustration that mass approximately traces light on large scales and that $\Omega_{m}$ is low, as suggested by Figure 1 .

\section{Baryons in Clusters}

Clusters contain many baryons, observed as gas and stars. Within $1.5 \mathrm{~h}^{-1}$ Mpc of a rich cluster, the X-ray emitting gas contributes $\sim 6 \mathrm{~h}^{-1.5 \%}$ of the cluster virial mass [16-18]. Stars contribute another $\sim 2 \%$. The baryon fraction observed in clusters is thus:

$$
\Omega_{b} / \Omega_{m} \gtrsim 0.06 \mathrm{~h}^{-1.5}+0.02
$$

The observed value represents a lower-limit to the baryon fraction since we count only the known baryons observed in gas and stars; additional baryonic matter may of course exist in the clusters. Standard Big Bang nucleosynthesis limits the baryon density of the universe to $[19,20]$ :

$$
\Omega_{b} \simeq 0.015 \mathrm{~h}^{-2}
$$

These facts suggest that the baryon fraction observed in rich clusters (eq. 1) exceeds that of an $\Omega_{m}=1$ universe $\left(\Omega_{b} /\left(\Omega_{m}=1\right) \simeq 0.015 \mathrm{~h}^{-2}\right.$; eq. 2$)$ by a factor of $\gtrsim 3$ (for $\mathrm{h} \gtrsim 0.5$ ). Since detailed hydrodynamic simulations [16,18] show that baryons do not segregate into rich clusters, the above results imply that either the mean density of the universe is lower than the critical density by a factor of $\gtrsim 3$, or that the baryon density is much larger than predicted by nucleosynthesis. The observed high baryonic mass fraction in clusters (eq. 1), combined with the nucleosynthesis limit (eq. 2), suggest (for $\mathrm{h} \simeq 0.65 \pm 0.1$ ):

$$
\Omega_{m} \lesssim 0.3
$$

This upper limit on $\Omega_{m}$ is a simple, model-independent and thus powerful constraint: a critical density universe is inconsistent with the high baryon 
fraction observed in clusters (assuming the nucleosynthesis limit on $\Omega_{b}$, eq. 2). [A universe dominated by hot dark matter that is too hot to clump into clusters may survive this critical test; however, such a hot universe is unable to form the observed galaxies at high redshift, and is therefore unlikely.]

\section{Evolution of Cluster Abundance}

The observed present-day abundance of rich clusters of galaxies places a strong constraint on cosmology: $\sigma_{8} \Omega_{m}{ }^{0.5} \simeq 0.5$, where $\sigma_{8}$ is the rms mass fluctuations on $8 \mathrm{~h}^{-1} \mathrm{Mpc}$ scale, and $\Omega_{m}$ is the present cosmological density parameter [21-26]. This constraint is degenerate in $\Omega_{m}$ and $\sigma_{8}$; models with $\Omega_{m}=1, \sigma_{8} \sim 0.5$ are indistinguishable from models with $\Omega_{m} \sim 0.25, \sigma_{8} \sim 1$. (A $\sigma_{8} \simeq 1$ universe is unbiased, with mass following light on large scales since galaxies (light) exhibits $\sigma_{8}$ (galaxies) $\simeq 1 ; \sigma_{8} \simeq 0.5$ implies a biased universe with mass distributed more diffusely than light).

The evolution of cluster abundance with redshift, especially for massive clusters, breaks the degeneracy between $\Omega_{m}$ and $\sigma_{8}$ [see, e.g. 23,24,27-34]. The evolution of high mass clusters is strong in $\Omega_{m}=1$, low- $\sigma_{8}$ (biased) Gaussian models, where only a very low cluster abundance is expected at $z>0.5$. Conversely, the evolution rate in low $-\Omega_{m}$ high- $\sigma_{8}$ models is mild and the cluster abundance at $z>0.5$ is much higher than in $\Omega_{m}=1$ models.

In low-density models, density fluctuations evolve and freeze out at early times, thus producing only relatively little evolution at recent times $(z \lesssim 1)$. In an $\Omega_{m}=1$ universe, the fluctuations start growing more recently thereby producing strong evolution in recent times; a large increase in the abundance of massive clusters is expected from $z \sim 1$ to $z \sim 0$. In a recent study by Bahcall, Fan, and Cen [31] we show that the evolution is so strong in $\Omega_{m}=1$ models that finding even a few Coma-like clusters at $z>0.5$ over $\sim 10^{3} \mathrm{deg}^{2}$ of sky contradicts an $\Omega_{m}=1$ model where only $\sim 10^{-2}$ such clusters would be expected (when normalized to the present-day cluster abundance). The evolution of the number density of Coma-like clusters was determined from observations using the CNOC cluster sample to $z<0.5$ and compared with cosmological simulations [30-32]. The data show only a slow evolution of the cluster abundance to $z \sim 0.5$, with $\sim 10^{2}$ times more clusters observed at these redshifts than expected for $\Omega_{m}=1$. The results yield $\Omega_{m} \simeq 0.3 \pm 0.1$.

The evolutionary effects increase with cluster mass and with redshift. The 
existence of the three most massive clusters observed so far at $z \sim 0.5-0.9$ places the strongest constraint yet on $\Omega_{m}$ and $\sigma_{8}$. These clusters (MS0016+16 at $z=0.55$, MS0451-03 at $z=0.54$, and MS1054-03 at $z=0.83$, from the Extended Medium Sensitivity Survey, EMSS [35,36]), are nearly twice as massive as the Coma cluster, and have reliably measured masses (including gravitational lensing masses, temperatures, and velocity dispersions; [34,3740]. These clusters posses the highest masses $\left(\gtrsim 8 \times 10^{14} \mathrm{~h}^{-1} \mathrm{M}_{\odot}\right.$ within 1.5 $\mathrm{h}^{-1}$ comoving Mpc radius), the highest velocity dispersions $\left(\gtrsim 1200 \mathrm{~km} \mathrm{~s}^{-1}\right)$, and the highest temperatures $(\gtrsim 8 \mathrm{keV})$ in the $z>0.5$ EMSS survey. The existence of these three massive distant clusters, even just the existence of the single observed cluster at $z=0.83$, rules out Gaussian $\Omega_{m}=1$ models for which only $\sim 10^{-5} z \sim 0.8$ clusters are expected instead of the 1 cluster observed (or $\sim 10^{-3} z>0.5$ clusters expected instead of the 3 observed). (See Bahcall \& Fan [34]).

In Figure 2 we compare the observed versus expected evolution of the number density of such massive clusters. The expected evolution is based on the Press-Schechter [41] formalism; it is presented for different $\Omega_{m}$ values (each with the appropriate normalization $\sigma_{8}$ that satisfies the observed present-day cluster abundance, $\left.\sigma_{8} \simeq 0.5 \Omega_{m}{ }^{-0.5} ;[23,26]\right)$. The model curves range from $\Omega_{m}=0.1\left(\sigma_{8} \simeq 1.7\right)$ at the top of the figure (flattest, nearly no evolution) to $\Omega_{m}=1\left(\sigma_{8} \simeq 0.5\right)$ at the bottom (steepest, strongest evolution). The difference between high and low $\Omega_{m}$ models is dramatic for these high mass clusters: $\Omega_{m}=1$ models predict $\sim 10^{5}$ times less clusters at $z \sim 0.8$ than do $\Omega_{m} \sim 0.2$ models. The large magnitude of the effect is due to the fact that these are very massive clusters, on the exponential tail of the cluster mass function; they are rare events and the evolution of their number density depends exponentially on their "rarity", i.e., depends exponentially on $\sigma_{8}^{-2} \propto \Omega_{m}[32,34]$. The number of clusters observed at $z \sim 0.8$ is consistent with $\Omega_{m} \sim 0.2$, and is highly inconsistent with the $\sim 10^{-5}$ clusters expected if $\Omega_{m}=1$. The data exhibit only a slow, relatively flat evolution; this is expected only in low $\Omega_{m}$ models. $\Omega_{m}=1$ models have a $\sim 10^{-5}$ probability of producing the one observed cluster at $z \sim 0.8$, and, independently, a $\sim 10^{-6}$ probability of producing the two observed clusters at $z \sim 0.55$. These results rule out $\Omega_{m}=1$ Gaussian models at a very high confidence level. The results are similar for models with or without a cosmological constant. The data provide powerful constraints on $\Omega_{m}$ and $\sigma_{8}: \Omega_{m}=0.2_{-0.1}^{+0.15}$ and $\sigma_{8}=1.2 \pm 0.3$ (68\% confidence level) [34]. The high $\sigma_{8}$ value for the mean mass fluctuations 
indicates a nearly unbiased universe, with mass approximately tracing light on large scales. This conclusion is consistent with the suggested flattening of the observed M/L ratio on large scales (Figure 1, section 2).

The rate of evolution of the cluster abundance depends strongly on $\sigma_{8}$, as was shown by Fan et al. [32]: $\operatorname{d} \operatorname{logn} / \mathrm{dz} \propto-\sigma_{8}^{-2}$; low $-\sigma_{8}$ models evolve exponentially faster than high $-\sigma_{8}$ models, for a given mass cluster. The strong exponential dependence arises because clusters of a given mass represent rarer density peaks in low $-\sigma_{8}$ models. We show [32] that the evolution rate at $\mathrm{z} \lesssim 1$ is relatively insensitive to the density parameter $\Omega_{m}$ or to the exact shape of the power spectrum. This is illustrated in Figure 3, where we present the cluster abundance ratio, $\mathrm{n}(\mathrm{z} \simeq 0.8) / \mathrm{n}(\mathrm{z} \simeq 0)$, as a function of $\sigma_{8}$ for all $\Omega_{m}$ values. The strong exponential evolution rate for low $-\sigma_{8}$ is clearly distinguished from the nearly no evolution expected for $\sigma_{8} \simeq 1$ (for any $\left.\Omega_{m}\right)$. The dependence on $\Omega_{m}$ is only secondary. This method thus provides a unique tool for determining $\sigma_{8}$ - by simply measuring the observed ratio of cluster abundance at different redshifts. When combined with the observed normalization of the present-day cluster abundance, $\sigma_{8} \Omega_{m}{ }^{0.5} \simeq 0.5$, the density parameter $\Omega_{m}$ can be determined. The observed evolution rate implied by the existence of the massive high redshift clusters discussed above is shown in Figure 3- it indicates only minimal evolution thus a high $\sigma_{8}$ value of $\sim 1$.

In Figure 4 we summarize the above results for $\Omega_{m}$ and $\sigma_{8}$ as determined from cluster evolution and compare the results with the other two independent methods discussed in sections 2 and 3. We show in Figure 4 the $\Omega_{m}-\sigma_{8}$ band allowed by the present-day cluster abundance $\Omega_{m}{ }^{0.5} \simeq 0.5 \sigma_{8}{ }^{-1}$, and the band allowed by the existence of the high-redshift massive clusters [34]. The intersection of these two bands provides the cluster evolution constraints discussed above; the only allowed range for Gaussian models is low $-\Omega_{m}, \sigma_{8} \sim 1$. We also present the $\Omega_{m}$ constraints determined from the two entirely independent methods discussed in $\S \S 2-3$ : the high baryon fraction observed in clusters, which yields $\Omega_{m} \lesssim 0.3$ (assuming the nucleosynthesis limit for the baryon density); and cluster dynamics which yields $\Omega_{m} \simeq 0.2$. All these independent methods yield consistent results: $\Omega_{m} \simeq 0.2 \pm 0.1$ and $\sigma_{8} \simeq 1.2 \pm 0.2$ (1 $\sigma$ level). $\Omega_{m}=1$ models are highly incompatible with these results. 


\section{Summary}

We have shown that several independent observations of clusters of galaxies all indicate that the mass-density of the universe is sub-critical: $\Omega_{m} \simeq 0.2 \pm 0.1$. The results also suggest that mass approximately traces light on large scales. A summary of the results, presented in Figure 4, is highlighted below.

1. The mass-to-light ratio of clusters (and superclusters) of galaxies and the suggested flattening of the mass-to-light ratio on large scales suggest $\Omega_{m} \simeq 0.2 \pm 0.1$.

2. The high baryon fraction observed in clusters of galaxies suggests $\Omega_{m} \lesssim 0.3$.

3. The weak evolution of the observed cluster abundance to $z \sim 1$ provides a robust estimate of $\Omega_{m} \simeq 0.2_{-0.1}^{+0.15}$, valid for any Gaussian models. An $\Omega_{m}=1$ Gaussian universe is ruled out as a $\lesssim 10^{-6}$ probability by the cluster evolution results (Figure 2-4).

4. All the above-described independent measures are consistent with each other and indicate a low-density universe with $\Omega_{m} \simeq 0.2 \pm 0.1$ (Figure 4). $\Omega_{m}=1$ models are ruled out by the data. While non-Gaussian initial fluctuations, if they exist, will affect the cluster evolution results, they will not affect arguments (1) and (2) above. Gaussian low-density models (with or without a cosmological constant) can consistently explain all the independent observations presented here. These independent cluster observations indicate that we live in a lightweight universe with only $\sim 20 \%-30 \%$ of the critical density. Thus, the universe may expand forever.

\section{Acknowledgments}

I am grateful to X. Fan and R. Cen with whom much of the work reviewed here was done. This work was supported in part by NSF grant AST93-15368.

\section{$7 \quad$ References}

1. Zwicky, F., "Morphological Astronomy" (Berlin: Springer-Verlag 1957).

2. Bahcall, N.A., Ann. Rev. Astron. Astrophys. 15, 505 (1977). 
3. Carlberg, R.G. et al., Astrophys. J. 462, 32 (1996).

4. Jones, C. \& Forman, W., Astrophys. J. 276, 38 (1984).

5. Sarazin, C.L., Rev. Mod. Phys. 58, 1 (1986).

6. Evrards, A.E., Metzler, C.A., \& Navarro, J.F., Astrophys. J. 469, 494 (1996).

7. Tyson, J.A., Wenk, R.A., \& Valdes, F. Astrophys. J. 349, L1 (1990).

8. Kaiser, N. \& Squires, G. Astrophys. J. 404, 441 (1993).

9. Smail, I., Ellis, R.S., Fitchett, M.J., \& Edge, A.C., Mon. Not. Roy. Astron. Soc. 273, 277 (1995).

10. Colley, W.N., Tyson, J.A., \& Turner, E.L, Astrophys. J. 461, L83 (1996).

11. Bahcall, N.A., Lubin, L., \& Dorman, V., Astrophys. J. 447, L81 (1995).

12. Ostriker, J.P., Peebles, P.J.E., \& Yahil, A., Astrophys. J. 193, L1 (1974).

13. Rubin, V.C., Proc. Natl. Acad. Sci. USA 90, 4814 (1993).

14. Kaiser, N., et al., astro-ph/9809268 (1998).

15. Bahcall, N.A. \& Fan, X., Proc. Nat. Academy of Sciences, USA 95, 5956 (1998).

16. White, S.D.M., Navarro, J.F., Evrard, A., \& Frenk, C.S., Nature 366, 429 (1993).

17. White, D. \& Fabian, A., Mon. Not. Roy. Astron. Soc. 272, 72 (1995).

18. Lubin, L., Cen, R., Bahcall, N.A., \& Ostriker, J.P., Astrophys. J. 460, 10 (1996).

19. Walker, T.P., et al., Astrophys. J. 376, 51 (1991). 
20. Tytler, D., Fan, X.-M., Burles, S., Nature 381, 207 (1996).

21. Bahcall, N.A. \& Cen, R., Astrophys. J. 398, L81 (1992).

22. White, S.D.M., Efstathiou, G., \& Frenk, C.S., Mon. Not. Roy. Astron. Soc. 262, 1023 (1993).

23. Eke, V.R., Cole, S., \& Frenk, C.S., Mon. Not. Roy. Astron. Soc. 282, 263 (1996).

24. Viana, P.P. \& Liddle, A.R., Mon. Not. Roy. Astron. Soc. 281, 323 (1996).

25. Kitayama, T. \& Suto, Y., Astrophys. J. 469, 480 (1996).

26. Pen, U.-L., Astrophys. J. 498, 60 (1998).

27. Peebles, P.J.E., Daly, R.A., \& Juszkiewicz, R., Astrophys. J. 347, 563 (1989).

28. Oukbir, J. \& Blanchard, A., Astron. Astrophys. 262, L21 (1992).

29. Oukbir, J. \& Blanchard, A., Astron. Astrophys. 317, 1 (1997).

30. Carlberg, R.G., Morris, S.M., Yee, H.K.C., \& Ellingson, E., Astrophys. J. 479, L19 (1997).

31. Bahcall, N.A., Fan, X., \& Cen, R., Astrophys. J. 485, L53 (1997).

32. Fan, X., Bahcall, N.A., \& Cen, R., Astrophys. J. 490, L123 (1997).

33. Henry, J.P., Astrophys. J. 489, L1 (1997).

34. Bahcall, N.A. \& Fan, X., Astrophys. J. 504, 1 (1998).

35. Henry, J.P, et al., Astrophys. J. 386, 408 (1992).

36. Luppino, G.A. \& Gioia, I.M., Astrophys. J. 445, L77 (1995).

37. Smail, I., Ellis, R.S., Fitchett, M.J., \& Edge, A.C., Mon. Not. Roy. Astron. Soc. 273, 277 (1995).

38. Luppino, G.A. \& Kaiser, N., Astrophys. J. 475, 20 (1997). 
39. Mushotsky, R. \& Scharf, C.A., Astrophys. J. 482, L13 (1997).

40. Donahue, M., et al., Astrophys. J. 502, 550 (1998).

41. Press, W.H. \& Schechter, P., Astrophys. J. 187, 425 (1974). 


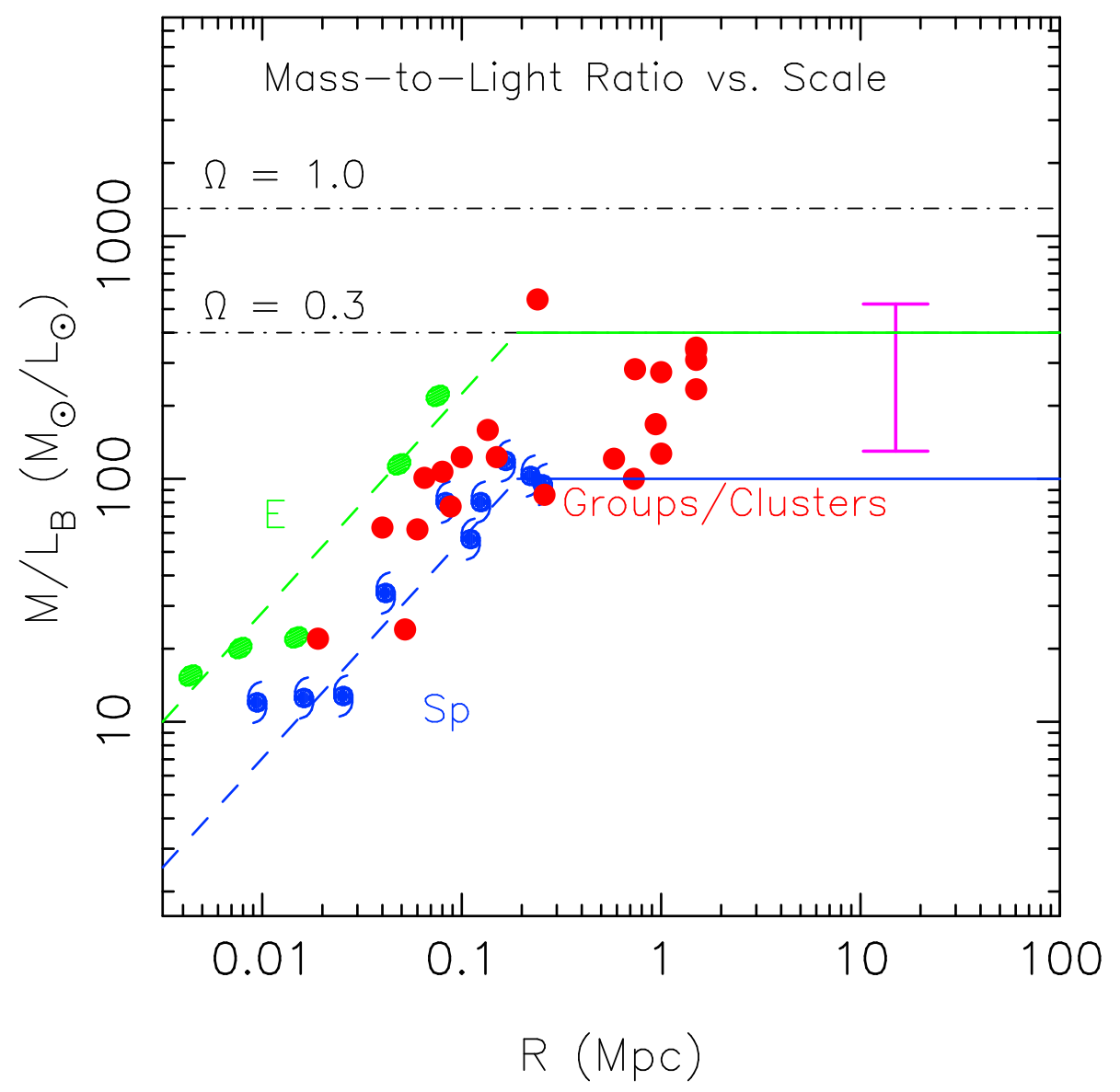

Figure 1: The dependence of mass-to-light ratio, $\mathrm{M} / \mathrm{L}_{\mathrm{B}}$, on scale, $\mathrm{R}$, for average spiral galaxies (spiral symbols), elliptical galaxies (elliptical symbols), and groups and clusters (filled circles). Adapted from Bahcall, Lubin and Dorman [11]; [15]. The large scale point at $\sim 15 \mathrm{~h}^{-1} \mathrm{Mpc}$ represents Virgo cluster infall motion results [11]. The location of $\Omega_{m}=1$ and $\Omega_{m}=0.3$ are indicated by the horizontal lines. A flattening of $\mathrm{M} / \mathrm{L}_{\mathrm{B}}$ is suggested at $\Omega_{m} \simeq 0.2 \pm 0.1$. A recent result for a supercluster using weak gravitational lensing $\left(\mathrm{R} \simeq 6 \mathrm{~h}^{-1} \mathrm{Mpc} ;[14]\right), \mathrm{M} / \mathrm{L}_{\mathrm{B}}=280 \pm 40 \mathrm{~h}$, is consistent with the suggested flattening of $\mathrm{M} / \mathrm{L}(\mathrm{R})$. 


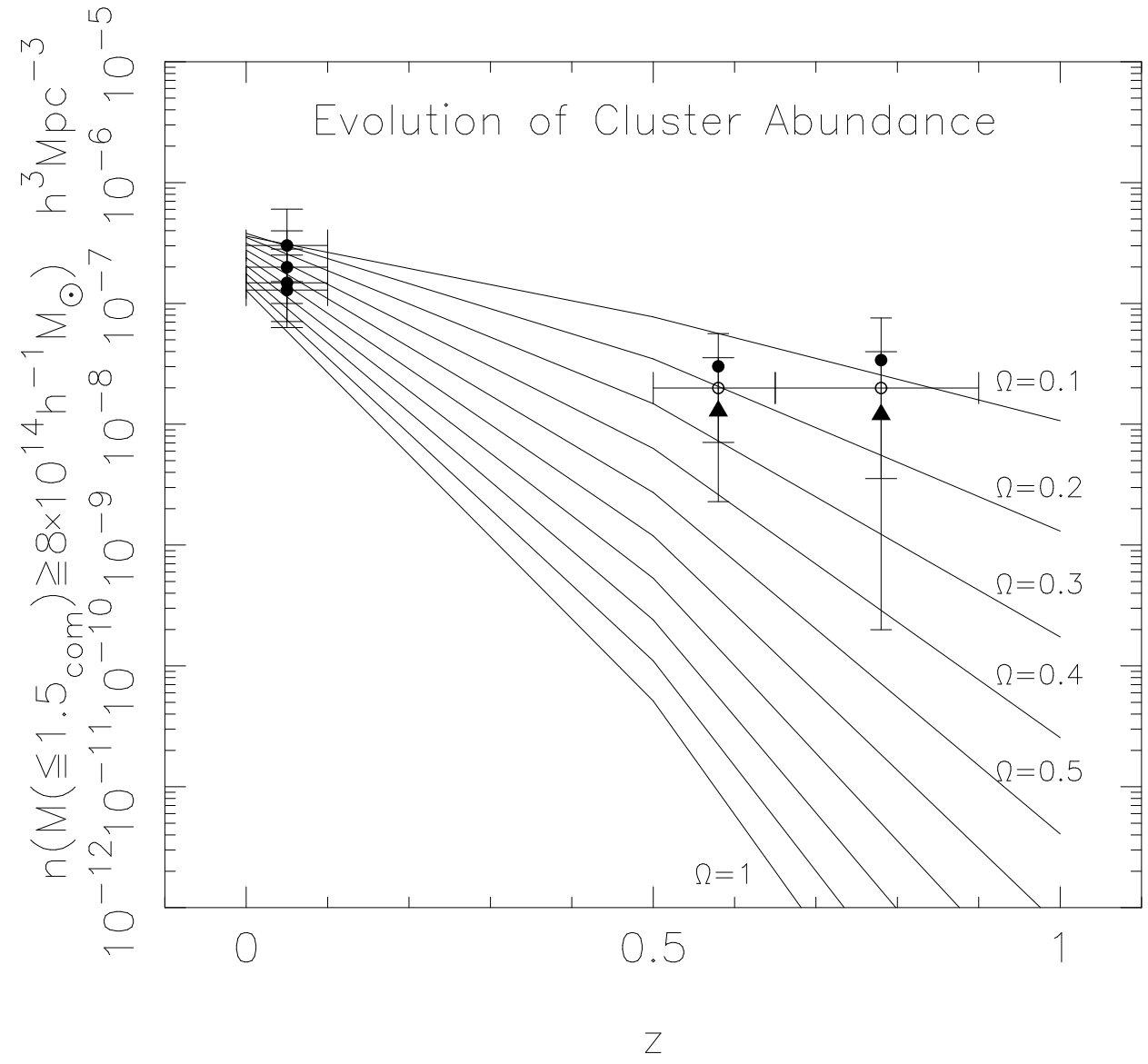

Figure 2: Evolution of the number density of massive clusters as a function of redshift: observed versus expected (for clusters with mass $\gtrsim 8 \times 10^{14} \mathrm{~h}^{-1} \mathrm{M}_{\odot}$ within a comoving radius of $1.5 \mathrm{~h}^{-1} \mathrm{Mpc}$ ). (Adapted from Bahcall and Fan 1998 [34]. The expected evolution is presented for different $\Omega_{m}$ values by the different curves. The observational data points (see text) show only a slow evolution in the cluster abundance, consistent with $\Omega_{m}$ $\simeq 0.2_{-0.1}^{+0.15}$. Models with $\Omega_{m}=1$ predict $\sim 10^{5}$ fewer clusters than observed at $z \sim 0.8$, and $\sim 10^{3}$ fewer clusters than observed at $z \sim 0.6$. 


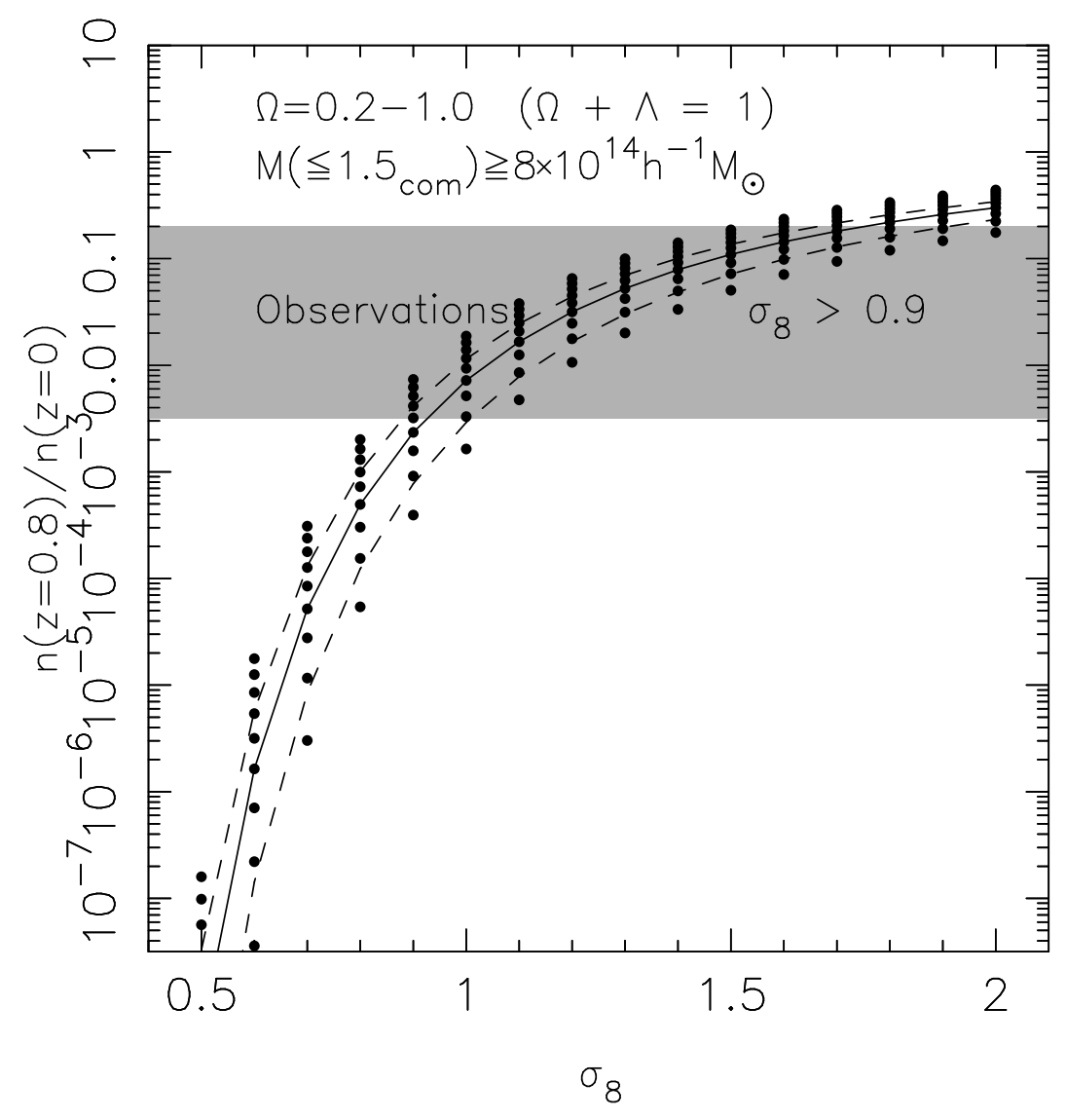

Figure 3: Cluster abundance ratio, $n(z=0.8) / n(z=0)$, vs. $\sigma_{8}$ from Press-Schechter (solid curve, for mean of all $\Omega_{m}$ 's) for clusters with mass $\gtrsim 8 \times 10^{14} \mathrm{~h}^{-1} \mathrm{M}_{\odot}$. Filled circles represent $\Omega_{m}$ 's from 0.2 to 1 (bottom to top). (Dashed curves represent the mass threshold range of 7 to $10 \times 10^{14} \mathrm{M}_{\odot}$, top and bottom, respectively). The data (Figure $2, \S 4$ ) are shown by the shaded region ( $68 \%$ level). Similar results are obtained for $\Lambda=0$. 


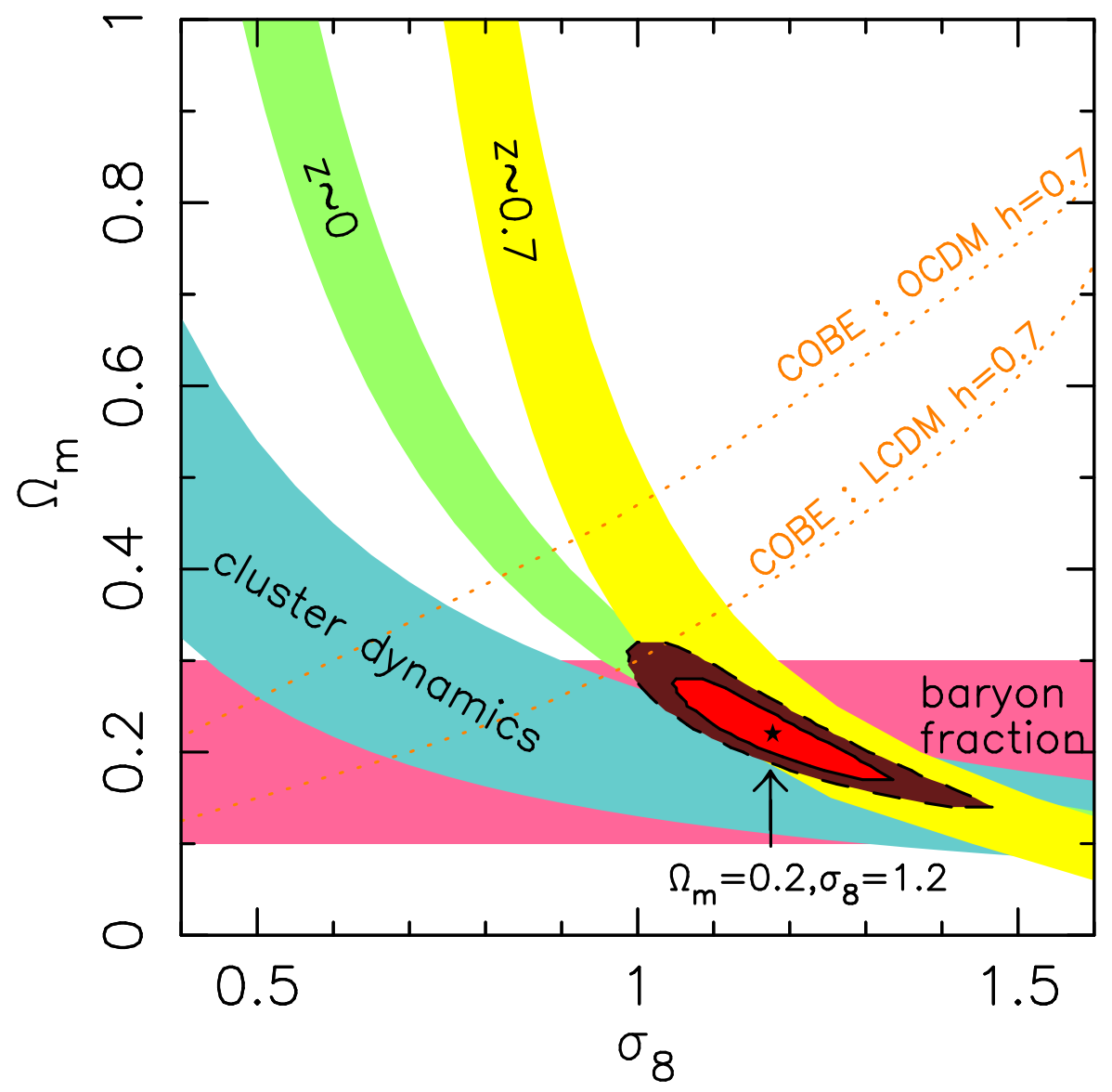

Figure 4: Constraining the mass-density parameter, $\Omega_{m}$, and the mass fluctuations on $8 \mathrm{~h}^{-1} \mathrm{Mpc}$ scale, $\sigma_{8}$, from several independent observations of clusters: cluster dynamics; baryon fraction in clusters; present-day cluster abundance $(z \sim 0)$; and cluster abundance at redshift $z \sim 0.7$. (The latter two abundances yield the cluster evolution constraints shown in Figure 2; see text). All these model-independent observations converge at the allowed range of $\Omega_{m}=0.2 \pm 0.1$ and $\sigma_{8}=1.2 \pm 0.2$ ( $68 \%$ confidence level). The dotted lines illustrate the mean microwave fluctuations constraints, based on the COBE satellite results, for a Cold-Dark-Matter model with $\mathrm{h}=0.7$ (with and without a cosmological constant, denoted as LCDM and OCDM respectively. Both models are consistent, within their uncertainties, with the best-fit $\Omega_{m}-\sigma_{8}$ regime of the cluster observations). 